\title{
De la participation en art
}

\author{
L'Écomusée du fier monde comme alliance entre l'art \\ et la participation citoyenne
}

Catherine Duchesneau

\begin{abstract}
L'art dit "participatif» est né récemment dans le champ de l'art, plaçant au centre du processus de création la participation de sujets. Cette nouvelle forme d'art n'est pas sans rappeler les grands fondements de la démocratie et ceux de la démocratie participative, laquelle renouvelle également la notion de participation. Or, comment l'art peut-il puiser dans les fondements de la démocratie tout en demeurant dans le champ de l'art? Cet article traitera du rapport qu'entretient l'art actuel avec le politique. Il tentera de renouveler la question de la frontière entre autonomie de l'art et instrumentalisation de l'art à des fins sociales ou politiques en présentant un cas d'alliance possible entre l'art et la participation citoyenne, soit l'Écomusée du fier monde. Mots-clés : Art, participation, art participatif, démocratie, démocratie participative, instrumentalisation de l'art.
\end{abstract}

\section{Introduction}

L'《art participatif», forme d'art au sein de laquelle la participation de sujets à l'œuvre d'art est le moteur de la création, a émergé récemment dans le champ de l'art, et plus précisément dans les arts visuels. Plaçant ainsi au centre du processus de création l'idée de participation, cette nouvelle forme de pratique artistique n'est pas sans rappeler le renouvellement qu'a connu le concept de démocratie avec l'émergence de celui de démocratie participative qui constitue une tentative de renouvellement d'un grand fondement de la démocratie, soit la participation du collectif (Dhoquois et M. Hatzfeld 2007). De la même façon, l'art participatif, et plus largement l'art relationnel, réactivent la participation en faisant intervenir des sujets dans la création même de l'œuvre, l'artiste partageant délibérément ses pouvoirs de création avec ces sujets participants. Or, cette forme d'art puisant dans des fondements démocratiques et situant au cœur du processus de création les liens créés entre l'artiste et le participant, la frontière entre l'art et le politique tend à être brouillée dans certaines œuvres d'art. Dans cet article, nous poserons donc la question suivante : si l'artiste (ou l'organisme artistique) place au centre de ses ouvres la participation citoyenne, celles-ci 
sont-elles pour autant instrumentalisées ? Tout en questionnant cette frontière entre l'art autonome et l'art instrumentalisé à des fins sociales ou politiques, nous nous décentrerons de cette stricte question binaire. Le cas de l'Écomusée du fier monde, organisme culturel montréalais, se présentera alors comme une tentative de renouvellement des termes de ce débat en ouvrant la voie à une autre possibilité, soit l'alliance entre l'art et la participation citoyenne. Combinant art participatif et démocratie participative, il illustrera un nouveau rapport au politique.

\section{De la participation dans l'art : l'art actuel et son rapport au politique}

Poser la question de l'instrumentalisation de l'art participatif à des fins sociales et politiques, c'est du même coup interroger le rapport entre l'art et le politique ainsi que la place de l'engagement politique dans les pratiques artistiques contemporaines. Et cette question est d'autant plus importante que de nouvelles formes d'art engagé ont changé les termes du débat. En effet, si les artistes occidentaux contemporains des années 1960-70, notamment ceux issus des arts visuels, prenaient position politiquement par l'entremise de manifestes, d'interventions dans le débat public et par des œuvres d'art à messages explicitement politiques, il en va autrement aujourd'hui (Lamoureux 2009). D'abord, l'engagement n'implique plus pour l'artiste d'être en marge des institutions ou de s'y confronter, ce qui peut par ailleurs être problématique puisque l'artiste semble sommé aujourd'hui, dans le contexte de démocratisation de la culture, de pratiquer un art citoyen, un art à vocation sociale (Jeudy 1999). Ainsi, nombre d'artistes tentent de rompre avec la conception de l'art comme objet élitiste ou, comme le dit Bourdieu (1979), comme objet de distinction. Ils tentent de démocratiser l'art, de rejoindre tous les publics, de le rendre accessible (Jeudy 1999). Aussi, il ne s'agit plus aujourd'hui pour les artistes de défendre de grandes utopies, mais bien de prendre position à des niveaux plus infimes, plus locaux, en somme, à des niveaux micropolitiques (Ardenne 1999), et d'entrer en rapport avec autrui. Ce nouveau rapport au politique se retrouve notamment dans les pratiques relationnelles en arts visuels, à l'intérieur desquelles nous incluons l'art participatif.

Précisons, en suivant Bourriaud (2001), que l'art relationnel, englobant toutes les pratiques qui placent la relation esthétique au cœur du procès artistique et abordant les thèmes notamment de la connexion, des rencontres, de la collaboration et des contrats, est à comprendre comme une critique du contexte postmoderne. Alors que la modernité politique exprimait la volonté d'émancipation des individus et des peuples, le progrès des techniques et l'amélioration des conditions de travail, la postmodernité exprimerait quant à elle les dérives ou les 
échecs de la première (Bourriaud 2001). Ainsi, en réaction à la postmodernité, décryptée par divers auteurs (Lyotard 1979 ; Sennett 1979; Lasch 2000) comme une fragmentation du monde, une crise du lien social, une rationalisation générale du processus de production et une montée en puissance de l'État, les pratiques artistiques sont aujourd'hui un terrain d'expérimentation du lien social et des rapports de proximité. Pour le dire autrement, l'œuvre d'art relationnelle se présente comme un interstice social, l'interstice étant selon Marx :

" [une] communauté d'échanges échappant au cadre de l'économie capitaliste, car soustraits à la loi du profit: troc, ventes à perte, productions autarciques, etc. [II] est un espace de relations humaines qui, tout en s'insérant plus ou moins harmonieusement et ouvertement dans le système global, suggère d'autres possibilités d'échanges que celles qui sont en vigueur dans ce système» (Bourriaud $2001: 16$ ).

Bien que l'art ait toujours été relationnel à des degrés divers, instituant un dialogue entre divers «correspondants et destinataires» (Bourriaud 2001 : 15) ou entre divers mondes, comme l'affirme Becker (1988), la transformation qui s'est récemment opérée au sein de la création artistique concerne le passage d'une relation établie entre l'humanité et les objets à une relation établie entre les sujets (artistes et participants à l'œuvre) (Roux, 2007 ; Milevska, 2006 ; Bourriaud, 2001).

La spécificité de l'art relationnel aujourd'hui se trouve ainsi dans l'accroissement de la relation entre les sujets dans la création même de l'œuvre, constituant un «terreau fertile » pour la forme d'art qui intéresse notre propos, soit l'art participatif (Roux 2007 : 2). Cette forme participative d'art englobe un large spectre de pratiques artistiques au sein desquelles la participation de sujets à une œuvre constitue la plaque tournante du processus de création. Précisons qu'il ne s'agit pas d'une simple interaction du public avec l'œuvre créée par l'artiste, mais bien plus encore de la participation de sujets à la création même de l'œuvre. Dans cette forme, l'artiste conçoit la structure permettant la participation sans aucune préconception quant au résultat final ; l'artiste fait intervenir des participants, lesquels ont un large pouvoir sur le devenir de l'œuvre. L'art participatif réactive en ce sens les fondements démocratiques de participation et de pouvoir du peuple puisque les artistes qui le pratiquent cèdent en grande partie le contrôle de l'œuvre aux participants (Bishop 2006). Plus précisément, un lien peut être établi entre l'art participatif et la définition de la démocratie donnée par Baechler, soit « un contrat ou un nœud de contrats, liant entre eux, selon des termes définis, des acteurs individuels et collectifs » (Baechler 1994 : 67). L'art participatif rejoint également le concept de démocratie participative, lequel s'est présenté dans les dernières années comme une façon de renouveler 
le concept de démocratie en rappelant la nécessaire participation des citoyens (Dhoquois et M. Hatzfeld 2007). Précisons que cette réanimation de la participation citoyenne au sein de la démocratie participative vise non seulement l'augmentation du pouvoir des citoyens, mais également la représentation de tous les intérêts, le dialogue entre les individus et les institutions, l'action à l'échelle locale et dans diverses sphères et l'éducation des citoyens (Cornwell 1990). Enfin, non seulement la notion de participation est centrale dans l'art participatif, mais cette forme d'art puise également dans ces principes de la démocratie participative, notamment dans le principe d'action à l'échelle locale et dans diverses sphères de la société (Cornwell 1990). Nous y reviendrons avec le cas de l'Écomusée du fier monde.

Or, si l'art participatif s'appuie sur des fondements démocratiques, n'y a-t-il pas danger d'instrumentalisation ? Précisons que la notion d'instrumentalisation renvoie ici strictement au fait de rendre utile l'art à diverses fins ou d'en faire un outil d'intervention, menaçant ainsi l'autonomie de l'art. Adorno (1974) affirmait d'ailleurs que la fonction sociale ou politique de l'art est précisément de ne pas en avoir :

«On peut douter que les œuvres d'art interviennent effectivement dans la politique ; lorsque cela se produit, c'est le plus souvent de façon périphérique et lorsqu'elles s'y efforcent, elles régressent généralement par rapport à leur concept. » (Adorno 1974: 307)

Aujourd'hui, selon Jeudy (1999) et Uzel (2002), c'est plus précisément l'injonction d'utilité sociale de l'inutile, soit l'art, qui est devenu problématique dans le rapport entre l'art et le politique. Or, si nous affirmons, à l'instar de ces auteurs, qu'une certaine séparation doit être préservée entre l'art et le politique, nous croyons par ailleurs que les termes du débat doivent être renouvelés. La présentation du cas de l'Écomusée du fier monde, qui puise à la fois dans l'art participatif et dans la démocratie participative, offrira l'exemple d'une combinaison possible entre art et participation citoyenne, permettant de sortir de la simple opposition binaire entre art autonome et art instrumentalisé.

\section{L'Écomusée du fier monde : une alliance entre art et participation citoyenne}

La question que nous posons est la suivante: si la participation citoyenne se trouve au cœur d'une œuvre artistique, cette œuvre est-elle pour autant instrumentalisée ? Les deux cas suivants illustreront brièvement l'opposition entre art autonome et art instrumentalisé afin de nous permettre par la suite de montrer 
en quoi le cas qui nous intéresse principalement, soit l'Écomusée du fier monde, allie différemment art et participation.

Le premier exemple est celui de l'œuvre intitulée Colin-maillard, de l'artiste québécoise Raphaëlle de Groot. Réalisé entre 1999 et 2001 en collaboration avec le centre Dare-Dare (centre de diffusion d'art multidisciplinaire de Montréal). Ce projet de dessin faisait intervenir la participation de neuf personnes non-voyantes et malvoyantes, lesquelles devaient chacune à leur tour se prêter à un exercice de dessin d'observation. "D'une main, [elles] devaient toucher des formes en pâte de sel et, de l'autre, transposer le parcours de leur exploration tactile sur une feuille de papier. Lors des entretiens, [de Groot] traçait le portrait de chacun des participants «à l'aveugle », sans regarder [sa] main travailler. » (de Groot 2001) À l'aide de techniques spéciales développées par deux organismes de Montréal, l'Institut Nazareth et Louis Braille, certaines esquisses étaient ensuite transposées en relief pour qu'elles soient lisibles au toucher. Dans cette œuvre d'art participatif, la participation constitue bel et bien le moteur du processus de création puisque l'artiste laisse dans une large mesure le devenir de l'œuvre entre les mains des participants. L'artiste n'est pas dictatrice; elle laisse un pouvoir aux participants. Aussi, bien que ce projet puisse avoir un impact social indirect, du fait qu'il institue un rapport avec la différence et amène l'art à l'extérieur de son milieu traditionnel, il demeure avant toute œuvre. L'œuvre d'art reste autonome puisqu'il ne s'agit pas d'intervenir socialement, au moyen de l'art, auprès de ces personnes marquées par une différence plus ou moins stigmatisante, mais d'accéder à une perception ou à un regard artistique autre. L'artiste explore en somme les contraintes du dessin "à l'aveugle» et la rencontre avec cet autre monde.

Le deuxième exemple est celui d'Action terroriste socialement acceptable. L'ATSA est un organisme québécois à but non lucratif fondé en 1998 par les artistes Pierre Allard et Annie Roy afin de «créer dans l'espace public des œuvres et événements transdisciplinaires sous forme d'interventions, d'installations, de performances ou de mises en scène réalistes motivées par le désir d'interpeller la population envers des causes sociales, environnementales et patrimoniales cruciales et préoccupantes» (ATSA 2010). La question de l'instrumentalisation de l'art se pose dans ce cas de façon importante puisque l'ATSA prône la production d'œuvres d'art comme «outil d'interaction et d'éducation populaire ». Bien que, tout comme l'œuvre de l'artiste précédente, les œuvres de l'ATSA placent principalement la participation de sujets au centre du processus de création, la frontière entre ce qui relève de l'art et ce qui relève des champs politiques et sociaux est ambiguë. Si l'art participatif, tel que nous l'avons mentionné précédemment, n'a pas a priori une utilité sociale ou politique, l'ATSA fait au contraire de ses œuvres des instruments d'intervention sociale et politique, enlevant à l'art ses fondements d'autonomie et d'inutilité. 
Alors que le principe de participation devrait être avant tout au service de l'œuvre, il devient un moyen de sensibilisation à divers problèmes, transformant du même coup les artistes de l'ATSA en intervenants sociaux. Il ne s'agit plus d'art participatif, mais bien de participation citoyenne au moyen de l'art.

Enfin, le cas de l'Écomusée du fier monde offre l'exemple d'une combinaison différente entre art et participation citoyenne puisqu'il réussit à allier les concepts d'art participatif et de démocratie participative sans toutefois les confondre. Cet organisme, situé dans le quartier populaire Centre-sud de Montréal, œuvre à la mise en valeur du patrimoine et de l'histoire ouvrière et industrielle de ce quartier. Il se distingue des deux exemples précédents puisqu'il rend possible une jonction entre art et participation citoyenne. D'abord, logé dans un ancien bain public, lequel servait dans les années 1930 de lieu d'hygiène à la population de ce quartier ouvrier, l'Écomusée du fier monde perpétue la vocation locale et collective de ce lieu en demeurant au service de la population du quartier. Aussi, sa fonction sociale et citoyenne, de même que ses fondements démocratiques, se retrouvent dans la notion même d'Écomusée puisque celui-ci est défini par le muséologue Georges-Henri Rivière comme " un instrument qu'un pouvoir et une population conçoivent, fabriquent et exploitent ensemble» (Binette 2009: 4). L'Écomusée du fier monde s'inscrit par ailleurs dans le mouvement de la Nouvelle muséologie, soit l'ouverture des musées à des populations et publics divers ou, pour le dire autrement, dans le mouvement de la démocratisation culturelle (Binette 2009). Il s'associe, depuis ses débuts, à divers groupes et organismes communautaires et culturels locaux. À cet effet, son directeur apporte une précision importante quant au rôle de l'institution :

«L'Écomusée ne fait pas d'alphabétisation, ni de travail social auprès de jeunes victimes de violence, il ne met pas sur pied des coopératives d'habitation et il n'intervient pas auprès des itinérants. Il ne dédouble pas ce qui existe déjà. Il travaille avec des experts du milieu et apporte comme contribution son expertise spécifique liée à la recherche et au processus d'une exposition. Il s'ajoute, avec ses moyens de musée, à une constellation d'organismes communautaires locaux. » (Binette 2009 : 15)

L'Écomusée du fier monde, contrairement à l'ATSA, distingue donc bien son mandat de celui des organismes communautaires et évite ainsi le glissement de sa fonction artistique et culturelle à une fonction d'intervention sociale ou politique.

L'Écomusée identifie trois niveaux de participation à ses activités. Le premier concerne la visite des expositions, laquelle est déjà un défi puisque la population visée, soit principalement les résidants de ce quartier ouvrier, fréquente peu les 
musées. Ce niveau de participation s'ancre donc dans une volonté de démocratisation culturelle et d'accroissement de l'interaction du public avec l'art et la culture. Le deuxième niveau concerne la participation de la population « en tant que source ", c'est-à-dire la participation à des entrevues visant à recueillir des éléments de mémoire pour la réalisation d'expositions. Par exemple, dans le cadre du projet sur l'histoire des femmes de quartiers ouvriers, «Entre l'usine et la cuisine », réalisé en 1984, les femmes participaient au projet en partageant leur histoire lors d'une entrevue, ainsi que leurs photos et objets personnels. Cette participation de la population permet de faire revivre une histoire correspondant de près à la réalité quotidienne et à la vie de travail dans un quartier ouvrier. Ce niveau de participation rejoint de près la notion d'art participatif définie précédemment puisque la population locale devient le moteur de la création de l'exposition. Le devenir de cette dernière repose sur l'histoire de ces femmes et sur leur apport. Il ne s'agit pas là seulement d'une interaction plus grande entre public et artiste, comme le vise le premier niveau de participation, mais bien de la construction d'une œuvre basée sur la participation des femmes du quartier. Enfin, dans un troisième niveau de participation, les participants réalisent la recherche ou le montage de l'exposition (Binette 2009). L'association entre l'Écomusée et l'organisme LOVE, lequel intervient auprès des jeunes victimes ou témoins de la violence dans les quartiers montréalais, offre un exemple de ce type de participation. Le projet Voix à entendre, réalité des jeunes (2009), issu de la collaboration entre ces deux organismes, combine l'objectif de sensibilisation des jeunes au phénomène de violence et l'expérimentation de la photographie, de la vidéo et des nouvelles technologies de communication comme moyen d'expression. Si LOVE s'occupe du volet intervention et sensibilisation, l'Écomusée organise des visites guidées du quartier pour alimenter la réflexion et l'inspiration et guide le montage de l'exposition des œuvres (Binette 2009). Ce type de projet constitue également une forme d'art participatif, puisqu'un large pouvoir créatif est laissé entre les mains des participants, mais à la différence du projet précédent, la visée sociale est centrale.

Peut-on parler dès lors d'une instrumentalisation de l'art ? Dans ce cas, bien qu'une ambigüité puisse exister, l'Écomusée s'en tient à sa fonction culturelle, tout comme dans le projet précédent, et l'organisme LOVE prend en charge l'intervention sociale et l'éducation populaire. Si ce projet a une utilité sociale certaine, l'Écomusée ne fait pour sa part que guider le processus artistique. Il ne s'agit pas clairement d'une instrumentalisation puisque les rôles de chacun restent distincts. La prochaine section tentera de montrer plus en profondeur en quoi l'Écomusée du fier monde sort de cette question de l'instrumentalisation de l'art puisqu'il allie, sans les confondre, les notions d'art participatif et de démocratie, instituant de cette façon un nouveau rapport au politique. 


\section{Pour un nouveau rapport au politique : la jonction entre art participatif et démocratie participative}

La participation citoyenne et l'éducation populaire étant centrales dans le mandat de l'Écomusée du fier monde, les projets de cet organisme ne correspondent pas strictement à l'art participatif, tel que nous l'avons défini et exemplifié, puisque ses projets ont une visée sociale et politique, contrairement au projet de Raphaëlle de Groot. Toutefois, l'Écomusée ne constitue pas non plus un cas d'instrumentalisation de l'art ou de participation citoyenne au moyen de l'art, puisque, contrairement à l'ATSA, il ne glisse pas vers une fonction d'intervention. Il s'associe à d'autres organismes afin que ces derniers assurent ce rôle, permettant à l'Écomusée de se consacrer uniquement à sa fonction culturelle. Ses fonctions citoyennes et artistiques (ou culturelles demeurent bien distinctes l'une de l'autre. L'Écomusée du fier monde combine donc à la fois des fondements de la démocratie participative et des fondements de l'art participatif (et de l'art relationnel) sans toutefois les confondre.

D'abord, l'Écomusée rejoint le concept de démocratie participative en réactivant la notion de participation citoyenne. Rappelons que cette réanimation de la participation citoyenne au sein de la démocratie participative vise non seulement l'augmentation du pouvoir des citoyens, mais également la représentation de tous les intérêts, le dialogue entre les individus et les institutions, l'action à l'échelle locale et dans diverses sphères et l'éducation des citoyens (Cornwell 1990). Loin d'être confinées au strict champ du politique, des expériences de démocratie participative, aux formes et résultats variés, intègrent divers milieux, tant associatifs et académiques, qu'institutionnels, gouvernementaux, artistiques ou corporatifs (Cornwell 1990). Ainsi, tout comme ces pratiques, l'Écomusée intervient localement, au sein d'un quartier populaire et ouvrier, et développe bon nombre de ses activités autour des missions de participation citoyenne, d'éducation populaire et d'empowerment. Aussi, se définissant comme un Écomusée, il fait dialoguer des milieux d'un même quartier qui n'entrent que rarement en interaction, soit le milieu de l'art ou de la culture et le milieu communautaire, et, par ses pratiques de participation citoyenne, il donne la parole à la population de ce quartier défavorisé. Enfin, il n'effectue pas une simple critique de la hiérarchie habituelle entre artiste et spectateurs, mais encore davantage une critique d'un plein pouvoir que détiendrait l'artiste sur la création, évoquant dès lors plus largement le sens étymologique de la démocratie, soit le pouvoir (kratia) du peuple (dèmos). En ce sens, il rappelle également la critique formulée par 
Tocqueville $\left(1961^{1}\right)$. Auteur de De la démocratie en Amérique, Tocqueville a été l'un des premiers à critiquer sévèrement la notion de démocratie, laquelle, selon lui, s'était transformée en un despotisme plutôt que de constituer, comme l'affirmait Rousseau $\left(1999^{2}\right)$, un contrat social garantissant liberté et égalité à l'ensemble des citoyens et devant faire primer l'intérêt collectif sur l'intérêt particulier.

Or, à la différence des pratiques de démocratie, les pratiques artistiques de l'Écomusée ont pour principal enjeu leur autonomie. Le processus de participation au sein de l'art participatif est effectivement avant tout au service de l'œuvre et, indirectement, lié à d'autres causes, tandis que dans les pratiques de démocratie participative, il est directement au service de causes diverses (sociales, politiques, urbaines, etc.). Conséquemment, le rôle des sujets des pratiques de démocratie participative et d'art participatif diffère en ce sens qu'au sein des premières, le sujet revêt son rôle de citoyen, tandis qu'au sein des secondes, il revêt un rôle de participant à l'œuvre. De la même façon, l'artiste qui initie la pratique artistique participative n'est pas investi de son rôle de citoyen, mais bien de son rôle d'artiste. Ainsi, si les participants aux projets de l'Écomusée sont réunis pour atteindre une finalité commune, cette finalité est, pour l'Écomusée, culturelle (ou artistique). La finalité sociale ou politique est prise en charge par les organismes avec qui l'Écomusée s'associe. C'est principalement grâce à cette distinction des rôles que l'Écomusée du fier monde évite un basculement de l'art autonome à l'art instrumentalisé à des fins sociales ou politiques, contrairement à l'ATSA. En d'autres mots, puisqu'il s'associe à des organismes communautaires, lesquels interviennent sur les questions sociales et de participation citoyenne, l'Écomusée peut se consacrer à son mandat artistique ou culturel. Le directeur de l'Écomusée (Binette 2009) affirme d'ailleurs ne pas dédoubler les activités des organismes communautaires auxquels il s'associe et demeurer un organisme à vocation culturelle « avec ses exigences de qualité ». Nous critiquons toutefois cette exigence de qualité puisque les expositions montées avec la participation des résidants du quartier, bien qu'encadrées par les professionnels de l'Écomusée, demeurent principalement des créations amateures locales, contrairement aux œuvres de Raphaëlle de Groot, par exemple, laquelle a acquis une renommée au sein de la discipline des arts visuels. Quoi qu'il en soit, le cas de l'Écomusée permet de réorienter la question de la frontière entre art autonome et de réfléchir sur un nouveau modèle

\footnotetext{
${ }^{1}$ Cet ouvrage de Tocqueville a été publié pour la première fois en 1835.
}

${ }^{2}$ Cet ouvrage de Rousseau a été publié pour la première fois en 1762 . 
d'alliance entre art participatif et démocratie participative puisqu'il fait cohabiter des visées artistiques et de participation citoyenne sans toutefois les confondre l'une dans l'autre.

\section{Conclusion}

En conclusion, le cas de l'Écomusée du fier monde a permis ultimement d'aborder l'engagement politique dans l'art actuel en exemplifiant les propos de divers auteurs, notamment Ardenne (1999) et Bourriaud (2001), lesquels affirment que la prise de position des artistes s'effectue aujourd'hui à des niveaux plus infimes, soit à des niveaux micropolitiques, et s'intéresse aux relations créées entre les sujets (artistes et participants). En abordant la façon dont certains fondements démocratiques s'expriment à l'intérieur de l'art participatif, il a été possible de comprendre ce nouveau rapport au politique, caractéristique des pratiques relationnelles en arts visuels. Par la présentation d'une expérience hybride liant art participatif et démocratie participative, cet article a pu par ailleurs renouveler le débat entre art autonome et art instrumentalisé. Malgré l'intérêt heuristique de ces deux catégories, l'émergence de nouvelles formes d'art montre bien que le débat ne se pose plus de façon binaire. D'ailleurs, dans le cas de l'Écomusée du fier monde, bien que la participation citoyenne soit centrale dans ses projets, en s'associant à des organismes communautaires locaux, cet organisme se consacre à sa fonction culturelle et artistique et évite ainsi le glissement vers une instrumentalisation de l'art. Or, en définitive, qu'elles soient instrumentalisées ou non, les pratiques qui lient art et participation portent toutes en elles le germe de la démocratie. Faisant dialoguer des mondes séparés, elles effectuent un retour au sens premier du politique, soit la participation aux affaires de la cité, et, plus encore, elles réactivent et actualisent la figure de l'agora grec comme lieu de débat public.

\section{Bibliographie}

Adorno, T. W. (1974), Théorie esthétique, Paris, Klincksieck.

Ardenne, P. (1999), L'Art dans son moment politique. Écrits de circonstance, Bruxelles, La Lettre volée.

ATSA (2010) Mandat et démarche. En ligne : http://www.atsa.qc.ca /pages/mandat.asp. Consultation le 23 novembre 2010.

Baechler, J. (1994), Précis de la démocratie, Paris, Calmann-Lévy/UNESCO.

Baudart, A. (2005), Qu'est-ce que la démocratie?, Paris, Librairie philosophie J. Vrin, coll. « Chemins philosophiques ». 
Becker, H. S. (1988), Les mondes de l'art, Paris, Flammarion.

Binette, R. (2009), «La contribution des institutions muséales au «capital social ». Le cas de l'Écomusée du fier monde (Montréal, Canada)», dans Activaciones patrimoniales e iniciativas museisticas : ? Por quien? Y? Para qué?, sous la dir. de Inaki Arrieta Urtizberea, Bilbao, Servicio Editorial de la Universitad del Pais Vasco.

Bishop, C. (2006), "The social turn. Collaboration and its discontents », Artforum, février 2006, p. 179-185.

Bourdieu, P. (1979), La distinction. Critique sociale du jugement, Paris, Éditions de Minuit.

Bourdieu, P. (1992), Les règles de l'art. Genèse et structure du champ littéraire, Paris, Éditions du Seuil.

Bourriaud, N. (2001), L'esthétique relationnelle, Dijon, Presses du réel.

Cornwell, T. L. (1990), Democracy and the arts. The role of participation, New York, Praeger Publishers.

De Groot, R. (2001), «L'autre comme contrée à explorer, Dévoilements et Colin-maillard », dans Les commensaux, Quand l'art se fait circonstances, p. 122129, sous la dir. de Patrice Loubier et Anne-Marie Ninacs, Montréal, Centre des arts actuels Skol.

Dhoquois, A. et M. Hatzfeld. (2007), Petites fabriques de la démocratie. Participer: idées, démarches, actions, Paris, Éditions Autrement.

Dumont, M. (2007), « Démocratisation », Espacetemps.net, 13.12.2007. En ligne : http:/ / espacestemps.net/document3903.html.

Jeudy, H.-P. (1999), Les usages sociaux de l'art, Belval, Circé.

Lamoureux, E. (2009), « Pratiques des artistes en arts visuels : un terrain fécond pour une réflexion sur les contours actuels de l'engagement », Revue canadienne de science politique, vol. 42, no 1, p. 45-63.

Lasch, C. (2000), La Culture du narcissisme. La vie américaine à un âge de déclin des espérances, Paris, Climats.

Lyotard, J.-F. (1979), La Condition postmoderne : rapport sur le savoir, Paris, Minuit, 1979.

Milevska, S. (2006), « Participatory art. A Paradigm Shift from Objects to Subjects ». Springerin, Issue 2/06. En ligne : 
http://www.springerin.at/dyn/heft.php?id=47\&pos=1\&textid=1761\&lang =en.

Mouffe, C. (2007), «Artistic Activism and Agonistic Spaces ». Art and research, vol. 1, no 1, été 2007.

Novitz, D. (2001), " Participatory art and Appreciate practice », The Journal of Aesthetics and Art Criticism, vol. 59, no 2, printemps 2001, p. 153-165.

Rousseau, J.-J. (1999), Du contrat social, livres I et II, Paris, Hatier.

Roux, X. (2007), " Participation in Contemporary Art », Colloque Supporting creative acts beyond dissemination. Creativity \& Cognition 2007, 13 juin, Washington DC.

Sennett, R. (1979), Les Tyrannies de l'intimité, Paris, Seuil.

Tocqueville, A. (1961), De la démocratie en Amérique, Paris, Gallimard.

Uzel, J.-P. (2002), "Art et politique dans les années 1990 », dans Identités narratives. Mémoire et perception, sous la dir. de Simon Harel, Jocelyne Lupien, Alexis Nouss et Pierre Ouellet, Québec, Les Presses de l'Université Laval, coll. « Intercultures », 2002, p.267-27 